\title{
DEMANDA ENERGÉTICA E EFICIÊNCIA DA DISTRIBUIÇÃO DE SEMENTES DE MILHO SOB VARIAÇÃO DE VELOCIDADE E CONDIÇÃO DE SOLO
}

\author{
DENISE MAHL ${ }^{1}$, CARLOS A. GAMERO ${ }^{2}$, SÉRGIO H. BENEZ ${ }^{2}$, CARLOS E. A. FURLANI ${ }^{3}$, \\ ANTÔNIO R. B. SILVA ${ }^{4}$
}

RESUMO: O trabalho teve o objetivo de avaliar a demanda energética e a eficiência da distribuição de sementes de uma semeadora-adubadora para semeadura direta, submetida à variação de velocidade e condições de solo, na semeadura da cultura do milho. O estudo foi desenvolvido em um Nitossolo Vermelho distrófico, na Fazenda Experimental Lageado, no município de Botucatu-SP. O delineamento experimental foi em blocos casualizados, com oito repetições, combinando-se três velocidades de deslocamento $\left(4,4 ; 6,1\right.$ e $\left.8,1 \mathrm{~km} \mathrm{~h}^{-1}\right)$ e duas condições de solo (solo manejado sob sistema de plantio direto há cinco anos e solo preparado com escarificador há 18 meses). Avaliaram-se a força de tração, a potência na barra de tração, o consumo de combustível, a capacidade de campo efetiva, a distribuição longitudinal de plantas, o coeficiente de variação, o índice de precisão e o número de plantas por hectare (estande inicial). Os resultados revelaram que, aumentando-se a velocidade de 4,4 para $8,1 \mathrm{~km} \mathrm{~h}^{-1}$, consegue-se aumentar em $86 \%$ a capacidade operacional, com incremento de $96 \%$ na demanda de potência na barra de tração e redução de $26 \%$ no consumo operacional de combustível. A maior velocidade $\left(8,1 \mathrm{~km} \mathrm{~h}^{-1}\right)$ proporcionou menor porcentual de espaçamentos normais e aumento no porcentual de espaçamentos múltiplos e falhos, maior coeficiente de variação e pior índice de precisão. A variação da velocidade não interferiu no número de plantas por hectare.

PALAVRAS-CHAVE: semeadura direta, demanda energética, distribuição de sementes.

\section{SEEDER ENERGETIC DEMAND AND DISTRIBUTION EFFICIENCY OF CORN SEEDS UNDER SPEED VARIATION AND SOIL CONDITION}

SUMMARY: This work evaluated the energetic demand and distribution efficiency of corn seeds in a no-till seeder when submitted to travel speed and soil condition variation. The study was developed in a soil classified as Ultissoil, at Fazenda Experimental Lageado, Botucatu Country, São Paulo State, Brazil. The experimental design was in a randomized block with eight repetitions, being the treatments a combination of three displacement speeds $\left(4.4 ; 6.1\right.$ and $8.1 \mathrm{~km} \mathrm{~h}^{-1}$ ) and two soil conditions (soil carried out under no tillage system during those last five years, soil prepared with chisel plow 18 months before seeding). Parameters of traction force, power, fuel consumption, effective field capacity, longitudinal distribution of plants, variation coefficient, precision index and initial plants stand were appraised. The results showed that increasing the speed of 4.4 to $8.1 \mathrm{~km} \mathrm{~h}^{-1}$, increases $86 \%$ the operational capacity of the group tractor/seeder, increasing $96 \%$ the power demand in the traction bar and reduction of $26 \%$ in the operational fuel consumption. The fastest speed $\left(8.1 \mathrm{~km} \mathrm{~h}^{-1}\right)$ provided a smaller percentile of normal spacing and an increasing in the percentile of multiple and fail spacing, larger variation coefficient and worse precision index. The speed variation didn't interfere in the plant stand.

KEYWORDS: no-tillage, energetic demand, seeds distribution.

\footnotetext{
${ }^{1}$ Engenheira Agrícola, aluna do Curso PG em Energia na Agricultura da FCA/UNESP - Câmpus de Botucatu, Botucatu - SP, Fone/Fax: (0XX14) 3811.7165, e-mail: denisemahl@fca.unesp.br

${ }^{2}$ Professor Titular, Departamento de Engenharia Rural, FCA/UNESP, Botucatu - SP.

${ }^{3}$ Professor Dr., Departamento de Engenharia Rural, FCAV/UNESP, Jaboticabal - SP.

${ }^{4}$ Aluno do Curso PG em Energia na Agricultura, FCA/UNESP - Câmpus de Botucatu, Botucatu - SP.

Recebido pelo Conselho Editorial em: 7-6-2002
}

Aprovado pelo Conselho Editorial em: 11-11-2003 


\section{INTRODUÇÃO}

A velocidade de trabalho e a heterogeneidade dos solos são fatores relevantes na avaliação da eficiência e do desempenho operacional de semeadoras-adubadoras. OLIVEIRA (1997) detectou que o aumento da velocidade provocou aumento significativo do consumo de combustível, o qual não foi influenciado por diferentes coberturas vegetais do solo, e, que a demanda de potência foi maior em solo Podzólico em relação ao Latossolo. SILVA (2000), variando a velocidade de semeadura de 3,1 a $8,7 \mathrm{~km} \mathrm{~h}^{-1}$ e diferentes mecanismos sulcadores para fertilizantes, verificou que houve influência da velocidade sobre o requerimento de força de tração e potência para a implantação da cultura de soja, sendo a maior demanda detectada na maior velocidade. No entanto, esse efeito não foi verificado na implantação da cultura de milho.

SIQUEIRA et al. (2001), avaliando quatro semeadoras-adubadoras na operação de semeadura direta de soja, detectaram aumento significativo no requerimento de força de tração e potência média e máxima na barra de tração, quando se aumentou a velocidade de deslocamento de 4,7 para 8,3 $\mathrm{km} \mathrm{h}^{-1}$. MARQUES et al. (1999), avaliando, em solo Nitossolo, a implantação da cultura do milho em sistemas de preparo de solo convencional e semeadura direta sob quatro diferentes métodos de manejo de vegetação espontânea, observaram que os tratamentos em semeadura direta não diferiram entre si quanto à capacidade de campo teórica, consumo de combustível, força de tração e potência média na barra.

DELAFOSSE (1986) afirmou que a velocidade de trabalho é um dos parâmetros que mais influencia no desempenho de semeadoras e que a distribuição longitudinal de sementes no sulco de semeadura é afetada pela velocidade de deslocamento, que, por sua vez, influencia na produtividade da cultura. KURACHI et al. (1986) relataram que as semeadoras-adubadoras de diferentes tipos e modelos existentes no mercado brasileiro devem ter sua eficiência avaliada por meio de dois parâmetros principais com relação à distribuição longitudinal de sementes, sendo eles a porcentagem de espaçamentos aceitáveis e o coeficiente de variação geral da produção de espaçamentos. Segundo KURACHI et al. (1989), estudos apontaram a uniformidade de distribuição longitudinal de sementes como uma das características que mais contribuem para um estande adequado de plantas e, conseqüentemente, para a melhoria da produtividade das culturas.

SILVA et al. (2000) conduziram um trabalho em solo com sistema de plantio direto por 12 anos para verificar o estabelecimento da cultura do milho, semeado por uma semeadora-adubadora equipada com dosador de sementes do tipo disco horizontal perfurado, nas velocidades periféricas de 3,0; 6,0; 9,0 e $11,2 \mathrm{~km} \mathrm{~h}^{-1}$ e profundidades de deposição de fertilizantes de 5 e $10 \mathrm{~cm}$. Os autores concluíram que o número de plantas na linha de semeadura foi reduzido com o aumento da velocidade. Por outro lado, FEY et al. (2000) afirmaram que o aumento da velocidade na operação de semeadura de milho influenciou na uniformidade de distribuição longitudinal de plantas, porém não afetou a população de plantas e a produtividade de grãos.

Avaliando três velocidades de deslocamento e dois mecanismos sulcadores em solo argiloso, MAHL et al. (2001) concluíram que o aumento da velocidade de deslocamento na operação de semeadura de milho reduziu o porcentual de espaçamentos aceitáveis e, conseqüentemente, aumentou o porcentual de espaçamentos múltiplos e falhos. Entretanto, SILVA (2000) concluiu que a uniformidade de distribuição de sementes não foi influenciada pela velocidade de deslocamento na implantação de culturas de milho e soja.

DAMBRÓS (1998) concluiu que a uniformidade de distribuição de plantas foi reduzida com o aumento da velocidade na operação de semeadura e verificou que a semeadora-adubadora pneumática apresentou maior porcentual de espaçamentos aceitáveis e menor coeficiente de variação na menor velocidade testada $\left(5,0 \mathrm{~km} \mathrm{~h}^{-1}\right)$. O autor acrescentou às análises o parâmetro "precisão de semeadura", 
o qual foi baseado na variabilidade de plantas em cada linha, correspondendo ao coeficiente de variação entre os espaçamentos aceitáveis. Observou que a semeadora com dosador pneumático, na velocidade de $5,0 \mathrm{~km} \mathrm{~h}^{-1}$, apresentou melhor precisão.

BONNIN ACOSTA (2000) avaliou, em condições de laboratório e de campo, semeadoras de covas para semeadura direta de milho, com diferentes configurações e mecanismos dosadores de sementes, considerando a variação de velocidade de 5,4; 7,2 e 9,0 km h${ }^{-1}$. Mediante análises de espaçamentos normais, múltiplos e falhos entre sementes, coeficiente de variação e precisão, o autor observou que tanto em condições de laboratório como de campo, o desempenho quanto aos parâmetros estudados foi melhor para a semeadora de cova com dosador pneumático na velocidade de $7,2 \mathrm{~km} \mathrm{~h}^{-1}$.

Problemas de desempenho de semeadoras-adubadoras de semeadura direta foram relacionados a solos com altos teores de argila. A alta resistência à penetração dos componentes rompedores nesses solos, associada a sua grande retenção de água, tem exigido constante adaptação das máquinas, especialmente na busca de soluções para problemas que afetam a uniformidade de emergência das plantas (CASÃO JÚNIOR et al., 1997).

O objetivo deste trabalho foi avaliar, em condições de campo, a demanda energética e a eficiência da distribuição de sementes de milho semeadas por uma semeadora-adubadora de precisão para semeadura direta submetida à variação de velocidade e condição de solo.

\section{MATERIAL E MÉTODOS}

O trabalho foi conduzido na Fazenda Experimental Lageado, pertencente à Faculdade de Ciências Agronômicas da UNESP, situada no município de Botucatu - SP, em solo classificado pela EMBRAPA (1999) como Nitossolo Vermelho distrófico. Implantou-se a cultura do milho (Zea mays L.), utilizando o híbrido triplo CO-32 (germinação de $85 \%$ e pureza de $98 \%$ ), o qual foi semeado sob restevas de triticale (Triticum turgidocereale) e de milho (safra 2000-2001), os quais apresentaram, em média, $9,77 \mathrm{t} \mathrm{ha}^{-1}$ de matéria seca. O teor médio de água no solo, na ocasião da semeadura foi de 30,0 e $30,3 \%$ nas camadas de 0 a 10 e 10 a $20 \mathrm{~cm}$, respectivamente.

O delineamento experimental foi em blocos casualizados, em esquema fatorial, com dois fatores: velocidades de deslocamento $\left(4,4 ; 6,1\right.$ e $\left.8,1 \mathrm{~km} \mathrm{~h}^{-1}\right)$ e condições de solo (solo sob sistema de plantio direto há cinco anos e solo sob efeito de escarificação), com oito repetições por tratamento. A operação de escarificação foi realizada a $30 \mathrm{~cm}$ de profundidade, 18 meses antes da realização deste trabalho. As velocidades foram selecionadas em função do escalonamento de marchas $(1 \mathrm{~B}, 2 \mathrm{~B}$ e $3 \mathrm{~B}$, respectivamente) do trator de marca John Deere, modelo 6600 , com $88,3 \mathrm{~kW}$ de potência no motor, utilizado na operação de semeadura. O experimento foi composto de 48 parcelas com dimensões de $20 \times 7 \mathrm{~m}$.

A semeadora-adubadora avaliada foi da marca Marchesan, modelo PST $^{2}$, equipada com seis unidades de semeadura e mecanismos sulcadores para adubo e sementes do tipo discos duplos defasados. A semeadora-adubadora foi regulada para efetuar a semeadura com espaçamento entre linhas de $45 \mathrm{~cm}$ e, entre sementes, de $35,09 \mathrm{~cm}$ (2,85 sementes por metro de linha semeada, perfazendo 63.333 sementes por hectare). O mecanismo dosador de sementes utilizado foi o disco horizontal perfurado, com 28 furos. Os depósitos de adubo e de sementes foram abastecidos a 50\% de sua capacidade.

As variáveis avaliadas foram força de tração, potência média na barra de tração, consumo de combustível, capacidade de campo efetiva, estande inicial de plantas e distribuição longitudinal de sementes. A distribuição longitudinal de sementes foi avaliada por meio de porcentuais de espaçamentos entre plantas normais, múltiplos e falhos, coeficiente de variação dos espaçamentos entre plantas e índice de precisão da distribuição de plantas de milho. 
Para a aquisição de dados, utilizou-se de um sistema de aquisição computadorizado "micrologger 21X, marca Campbell Scientific", para monitorar e exibir os dados da célula de carga e do gerador de impulso (fluxômetro). Os dados foram armazenados continuamente em um módulo de armazenamento externo de dados "Storage module SM196". O monitoramento da velocidade foi feito indiretamente por meio da freqüência de aquisição de dados de $20 \mathrm{~Hz}$ do "micrologger 21X". Para a determinação da força de tração requerida pela semeadora-adubadora, utilizou-se de uma célula de carga de marca Sodmex, modelo N-400, com capacidade de $50 \mathrm{kN}$. Para quantificar o consumo de combustível, utilizou-se de um fluxômetro, marca "Flowmate" oval, modelo Oval M-III, o qual emitiu ao sistema de aquisição de dados uma unidade de pulso a cada $\mathrm{mL}$ de combustível que passou pelo mesmo. O consumo operacional de combustível foi determinado por meio da relação entre o consumo horário de combustível e a capacidade de campo efetiva. A potência média exigida na barra de tração foi obtida por meio do produto entre a força e a velocidade média. A capacidade de campo efetiva foi determinada pela relação entre a área útil da parcela trabalhada e o tempo gasto no percurso da parcela.

A regularidade de distribuição longitudinal de plantas na linha de semeadura foi determinada aos 21 dias após a semeadura, procedendo-se à mensuração da distância entre as plantas de milho existentes em quatro linhas de cinco metros $\left(9 \mathrm{~m}^{2}\right)$. Os espaçamentos entre plantas $\left(\mathrm{X}_{\mathrm{i}}\right)$ foram analisados mediante classificação adaptada de KURACHI et al. (1989) para avaliação de espaçamentos entre sementes, determinando-se o porcentual de espaçamentos correspondentes às classes: normais $\left(\mathrm{X}_{\mathrm{ref}}<\mathrm{X}_{\mathrm{i}}<1,5 \mathrm{X}_{\mathrm{ref}}\right)$, múltiplos $\left(\mathrm{X}_{\mathrm{i}}<0,5 \mathrm{X}_{\mathrm{ref}}\right)$ e falhos $\left(\mathrm{X}_{\mathrm{i}}>1,5 \mathrm{X}_{\mathrm{ref}}\right)$, baseado em espaçamento de referência $\left(\mathrm{X}_{\mathrm{ref}}\right)$, de acordo com a regulagem da semeadora-adubadora. Dessa forma, os espaçamentos entre plantas foram classificados como normais $\left(17,55 \mathrm{~cm}<\mathrm{X}_{\mathrm{i}}<52,64 \mathrm{~cm}\right)$, múltiplos $\left(\mathrm{X}_{\mathrm{i}}<17,55 \mathrm{~cm}\right)$ e falhos $\left(\mathrm{X}_{\mathrm{i}}>52,64 \mathrm{~cm}\right)$, baseados no espaçamento de referência teórico regulado para a semeadora-adubadora $(35,09 \mathrm{~cm})$ utilizada. $\mathrm{O}$ estande inicial foi determinado por meio da contagem das plantas contidas na área de amostragem da determinação da distribuição longitudinal de plantas, sendo os valores transformados e expressos em número de plantas por hectare.

Para expressar a regularidade dos espaçamentos entre plantas, determinou-se o coeficiente de variação de todos os espaçamentos entre plantas da amostragem (normais, múltiplos e falhos), por meio da equação:

$$
\mathrm{CV}=\frac{\mathrm{S}_{2}}{\mathrm{X}} 100
$$

em que,

CV - coeficiente de variação, \%;

$\mathrm{S}_{2}$ - desvio-padrão de todos os espaçamentos entre plantas, $\mathrm{cm}$, e

$\mathrm{X}$ - média de todos os espaçamentos entre plantas, $\mathrm{cm}$.

O índice de precisão de distribuição de plantas correspondeu à relação entre o desvio-padrão dos espaçamentos normais e o espaçamento de referência da semeadora-adubadora utilizada, calculado pela equação:

$$
\mathrm{IP}=\frac{\mathrm{S}_{2}}{\mathrm{X}_{\text {ref }}} 100
$$

em que,

IP - índice de precisão;

$\mathrm{S}_{2}$ - desvio-padrão dos espaçamentos normais entre plantas, $\mathrm{cm}, \mathrm{e}$

$\mathrm{X}_{\text {ref }}$ - espaçamento de referência, $35,09 \mathrm{~cm}$. 
As médias dos dados obtidos foram submetidas à análise de variância e, nas causas de variações significativas $(\mathrm{P}<0,05)$, procedeu-se ao teste de Tukey, a $5 \%$ de significância, para comparar os contrastes entre médias. Determinaram-se, também, valores de coeficiente de variação.

\section{RESULTADOS E DISCUSSÃO}

$\mathrm{Na}$ Tabela 1, são apresentados os resultados de força de tração, potência média na barra de tração, consumo de combustível e capacidade de campo efetiva na operação de semeadura do milho.

TABELA 1. Análise de variância expressa pelo Teste $\mathrm{F}$ e teste de médias para as variáveis força de tração - FT, potência na barra de tração - PB, consumo operacional de combustível COC, e capacidade de campo efetiva - CCE.

\begin{tabular}{lcccc}
\hline \multirow{2}{*}{ Fatores } & \multicolumn{5}{c}{ Variáveis } \\
\cline { 2 - 5 } Condição do solo (P) & FT $(\mathrm{kN})$ & PB $(\mathrm{kW})$ & $\mathrm{COC}\left(\mathrm{L} \mathrm{ha}^{-1}\right)$ & $\mathrm{CCE}\left(\mathrm{ha} \mathrm{h}^{-1}\right)$ \\
Plantio direto & $8,22 \mathrm{a}$ & $14,27 \mathrm{a}$ & $7,08 \mathrm{a}$ & $1,68 \mathrm{a}$ \\
Escarificado & $8,44 \mathrm{a}$ & $14,63 \mathrm{a}$ & $7,26 \mathrm{a}$ & $1,67 \mathrm{a}$ \\
\hline Velocidade (V) & & & & \\
$4,4 \mathrm{~km} \mathrm{~h}^{-1}$ & $8,20 \mathrm{~b}$ & $9,93 \mathrm{c}$ & $8,43 \mathrm{a}$ & $1,18 \mathrm{c}$ \\
$6,1 \mathrm{~km} \mathrm{~h}^{-1}$ & $8,16 \mathrm{~b}$ & $13,92 \mathrm{~b}$ & $6,49 \mathrm{~b}$ & $1,66 \mathrm{~b}$ \\
$8,1 \mathrm{~km} \mathrm{~h}^{-1}$ & $8,64 \mathrm{a}$ & $19,51 \mathrm{a}$ & $6,24 \mathrm{c}$ & $2,19 \mathrm{a}$ \\
\hline Teste F & & & & \\
$\quad \mathrm{P}$ & $3,03 \mathrm{~ns}$ & $2,10 \mathrm{~ns}$ & $3,75 \mathrm{~ns}$ & $0,05 \mathrm{~ns}$ \\
$\quad \mathrm{~V}$ & $5,90 *$ & $501,81 *$ & $199,83 *$ & $2.593,22 *$ \\
P x V & $1,14 \mathrm{~ns}$ & $1,30 \mathrm{~ns}$ & $2,16 \mathrm{~ns}$ & $0,64 \mathrm{~ns}$ \\
\hline C.V. $(\%)$ & 5,31 & 5,94 & 4,45 & 2,38 \\
\hline
\end{tabular}

Médias seguidas de letras diferentes, em cada coluna e para cada fator, diferem entre si, pelo Teste de Tukey a $5 \%$ de probabilidade; * - representa efeito significativo $(\mathrm{P}<0,05)$; ns - representa o efeito não significativo $(\mathrm{P}>0,05)$, e C.V. corresponde ao coeficiente de variação.

Analisando-se a variável força de tração na barra, observou-se que essa não foi influenciada pela condição de preparo do solo. Entretanto, com relação à velocidade de deslocamento, a força de tração nas duas velocidades menores $\left(4,4\right.$ e $\left.6,1 \mathrm{~km} \mathrm{~h}^{-1}\right)$ foi semelhante, e essas diferiram da maior velocidade testada $\left(8,1 \mathrm{~km} \mathrm{~h}^{-1}\right)$. De acordo com HARRIGAN e ROTZ (1994), a força de tração na operação de semeadura não é função da velocidade de deslocamento. Ressalta-se, no entanto, que seus estudos foram conduzidos em solos com características e propriedades diferentes dos solos brasileiros, fato que pode justificar a ocorrência de tal variação.

As condições de solo não interferiram no requerimento de potência na barra de tração. Por outro lado, houve aumento gradativo da potência com o aumento da velocidade de deslocamento, de forma que, aumentando-se a velocidade de 4,4 para $8,1 \mathrm{~km} \mathrm{~h}^{-1}$, houve um aumento de $96 \%$ no requerimento de potência. Resultados semelhantes foram encontrados por OLIVEIRA (1997) e SIQUEIRA et al. (2001). SILVA (2000) também obteve maior demanda de potência na maior velocidade e verificou que o aumento da velocidade de 5,2 para $8,4 \mathrm{~km} \mathrm{~h}^{-1}$ aumentou a demanda de potência em $67 \%$.

Verificou-se que as variáveis consumo operacional de combustível $\left(\mathrm{L} \mathrm{ha}^{-1}\right)$ e capacidade de campo efetiva não foram influenciadas pela condição de solo. Quanto à variação de velocidade de deslocamento do conjunto trator/semeadora-adubadora, houve efeito da mesma sobre o consumo de combustível e a capacidade de campo efetiva. À medida que se aumentou a velocidade, houve redução significativa do consumo operacional de combustível e aumento da capacidade de campo efetiva. Por meio do aumento da velocidade de 4,4 para $8,1 \mathrm{~km} \mathrm{~h}^{-1}$, conseguiu-se um incremento de $86 \%$ na 
capacidade operacional do conjunto com redução de $26 \%$ no consumo operacional de combustível. Comportamento semelhante também foi encontrado por MAHL \& GAMERO (2003).

Observou-se, ainda, que, para todas as variáveis de demanda energética estudadas, o coeficiente de variação foi relativamente baixo, e o maior valor de 5,94\% ocorreu para a variável potência média requerida na barra de tração.

$\mathrm{Na}$ Tabela 2, são apresentados os resultados de estande inicial de plantas, da distribuição longitudinal de plantas de milho (expressa pelos porcentuais de espaçamentos normais, múltiplos e falhos), dos coeficientes de variação e dos índices de precisão.

TABELA 2. Análise de variância expressa pelo Teste $\mathrm{F}$ e teste de médias para as variáveis estande inicial de plantas - EI, porcentual de espaçamentos normais entre plantas - EN, porcentual de espaçamentos múltiplos - EM, porcentual de espaçamentos falhos - EF, coeficiente de variação na distribuição de plantas - CV, e índice de precisão na distribuição de plantas de milho - IP.

\begin{tabular}{|c|c|c|c|c|c|c|}
\hline \multirow{2}{*}{ Fatores } & \multicolumn{6}{|c|}{ Variáveis } \\
\hline & EI (plantas ha-1) & EN (\%) & EM (\%) & $\mathrm{EF}(\%)$ & C.V. $(\%)$ & IP \\
\hline \multicolumn{7}{|l|}{ Condição do solo (P) } \\
\hline Plantio direto & $59.167 \mathrm{a}$ & 85,85 a & $2,33 \mathrm{a}$ & 11,82 a & 33,93 a & 18,32 a \\
\hline Escarificado & $59.861 \mathrm{a}$ & 86,59 a & $2,76 \mathrm{a}$ & $10,65 \mathrm{a}$ & 33,29 a & $19,10 \mathrm{a}$ \\
\hline \multicolumn{7}{|l|}{ Velocidade (V) } \\
\hline $4,4 \mathrm{~km} \mathrm{~h}^{-1}$ & $60.139 \mathrm{a}$ & 88,39 a & $1,94 \mathrm{~b}$ & $9,66 \mathrm{~b}$ & 29,92 b & $16,05 \mathrm{~b}$ \\
\hline $6,1 \mathrm{~km} \mathrm{~h}^{-1}$ & $60.000 \mathrm{a}$ & 88,56 a & $1,50 \mathrm{~b}$ & $9,94 \mathrm{~b}$ & $32,00 \mathrm{~b}$ & $19,30 \mathrm{a}$ \\
\hline $8,1 \mathrm{~km} \mathrm{~h}^{-1}$ & $58.403 \mathrm{a}$ & $81,70 \mathrm{~b}$ & $4,19 \mathrm{a}$ & $14,11 \mathrm{a}$ & 38,91 a & $20,78 \mathrm{a}$ \\
\hline \multicolumn{7}{|l|}{ Teste F } \\
\hline $\mathrm{P}$ & $0,73 \mathrm{~ns}$ & $0,24 \mathrm{~ns}$ & $0,43 \mathrm{~ns}$ & $0,80 \mathrm{~ns}$ & $0,10 \mathrm{~ns}$ & $1,11 \mathrm{~ns}$ \\
\hline V & $1,87 \mathrm{~ns}$ & $8,95 *$ & $6,68 *$ & $4,85 *$ & $6,77 *$ & $14,23 *$ \\
\hline $\mathrm{P} \times \mathrm{V}$ & $1,29 \mathrm{~ns}$ & $0,28 \mathrm{~ns}$ & $0,09 \mathrm{~ns}$ & $0,36 \mathrm{~ns}$ & $0,12 \mathrm{~ns}$ & $1,55 \mathrm{~ns}$ \\
\hline C.V. (\%) & 4,74 & 6,07 & 87,64 & 40,28 & 21,52 & 13,74 \\
\hline
\end{tabular}

Médias seguidas de letras diferentes, em cada coluna e para cada fator, diferem entre si, pelo Teste de Tukey, a $5 \%$ de probabilidade; * - representa efeito significativo $(\mathrm{P}<0,05)$; ns - representa o efeito não significativo $(\mathrm{P}>0,05)$, e C.V. corresponde ao coeficiente de variação.

De acordo com a Tabela 2, os tratamentos em estudo não interferiram no estande inicial de plantas; no entanto, houve redução no estande $(6 \%)$ em relação à quantidade de sementes prevista por meio da regulagem da semeadora-adubadora. Resultados semelhantes foram encontrados por SILVA et al. (2000).

Com relação às variáveis de regularidade de distribuição de sementes, o fator condição de solo não interferiu no porcentual de espaçamentos normais, múltiplos e falhos, no coeficiente de variação entre os espaçamentos e no índice de precisão da distribuição de plantas de milho. Por outro lado, a velocidade de deslocamento na operação de semeadura da cultura do milho interferiu na distribuição longitudinal de plantas, no coeficiente de variação e no índice de precisão da distribuição das plantas. As velocidades de semeadura de 4,4 e $6,1 \mathrm{~km} \mathrm{~h}^{-1}$ apresentaram o mesmo desempenho com relação ao porcentual de espaçamentos normais, múltiplos e falhos, e ao coeficiente de variação. Tal desempenho foi significativamente melhor ao obtido na velocidade de $8,1 \mathrm{~km} \mathrm{~h}^{-1}$. FEY et al. (2000) e MAHL et al. (2001) também observaram que a variação de velocidade interferiu na distribuição longitudinal de plantas. 
Observou-se grande variabilidade entre os valores de porcentual de espaçamentos múltiplos (coeficiente de variação de 87,64\%). Em média, a freqüência de ocorrência de espaçamentos falhos foi 4,4 vezes maior que de espaçamentos múltiplos, fato esse associado à redução do estande inicial de plantas (em relação ao esperado pela regulagem da máquina). Por outro lado, obtiveram-se, em média, $86 \%$ de espaçamentos normais entre plantas, resultado considerado satisfatório quando comparado aos obtidos por DAMBRÓS (1998), BONNIN ACOSTA (2000) e MAHL et al. (2001). Ressalta-se, entretanto, que, devido à realização do experimento com unidades de semeadura espaçadas de $0,45 \mathrm{~m}$, o espaçamento entre as sementes foi maior, o que, provavelmente, permitiu a obtenção de maior porcentual de espaçamentos normais, uma vez que se considera o intervalo de 0,5 a 1,5 vez o espaçamento desejado.

A maior variabilidade na distribuição de sementes, expressa pelo coeficiente de variação de $38,91 \%$, foi obtida na maior velocidade testada $\left(8,1 \mathrm{~km} \mathrm{~h}^{-1}\right)$. DAMBRÓS (1998) também encontrou menor coeficiente de variação para a menor velocidade de semeadura, e BONNIN ACOSTA (2000) encontrou valores de coeficiente de variação acima de $45 \%$ quando variou a velocidade. A avaliação do índice de precisão revelou que a velocidade de $4,4 \mathrm{~km} \mathrm{~h}^{-1}$, diferiu estatisticamente das demais, apresentando a melhor eficiência na distribuição de sementes, isto é, nessa velocidade, ocorreu a maior freqüência de espaçamentos cujos valores foram próximos do espaçamento desejado $(35,09 \mathrm{~cm})$.

A ausência de efeito do fator condição de solo sobre qualquer variável estudada sugere que o efeito do preparo do solo com uso de escarificador a $30 \mathrm{~cm}$ de profundidade, após o período de 18 meses sob sistema de plantio direto, neutralizou-se, ou ambos os sistemas de preparo de solo proporcionam o mesmo desempenho de semeadoras-adubadoras com relação à demanda energética e à eficiência de distribuição de plantas de milho.

\section{CONCLUSÕES}

Não houve efeito residual do preparo de solo com escarificador após o período de 18 meses sobre as variáveis estudadas. A velocidade de deslocamento na operação de semeadura interferiu no desempenho do conjunto trator/semeadora com relação à demanda energética e capacidade de campo efetiva. O aumento da velocidade de 4,4 para $8,1 \mathrm{~km} \mathrm{~h}^{-1}$ proporcionou aumento de $86 \%$ na capacidade operacional do conjunto, com incremento de $96 \%$ na demanda de potência na barra de tração e redução de $26 \%$ no consumo operacional de combustível.

Nas velocidades de semeadura de 4,4 e $6,1 \mathrm{~km} \mathrm{~h}^{-1}$, obteve-se eficiência semelhante na distribuição de sementes de milho e significativamente melhor que na velocidade de $8,1 \mathrm{~km} \mathrm{~h}^{-1}$. A maior velocidade proporcionou menor porcentual de espaçamentos normais e aumento no porcentual de espaçamentos múltiplos e falhos, maior coeficiente de variação e pior índice de precisão. A melhor precisão na distribuição de sementes ocorreu na menor velocidade de deslocamento. A variação da velocidade não interferiu no estande inicial de plantas.

\section{REFERÊNCIAS BIBLIOGRÁFICAS}

BONNIN ACOSTA, J.J. Avaliação de diferentes protótipos de semeadoras em covas para semeadura direta de milho. 2000. 84 f. Dissertação (Mestrado em Máquinas Agrícolas) - Escola Superior de Agricultura “Luiz de Queiroz”, Universidade de São Paulo, Piracicaba, 2000.

CASÃO JÚNIOR, R.; PALLEROSI, C.A.; PORTELLA, J.A. Dispositivo pneumático para redução dos erros de dosagem e deposição de sementes. In: CONGRESSO BRASILEIRO DE ENGENHARIA AGRÍCOLA, 26., 1997, Campina Grande. Anais... Campina Grande: Sociedade Brasileira de Engenharia Agrícola, 1997. 1 CD.

DAMBRÓS, R.M. Avaliação do desempenho de semeadoras-adubadoras de milho com diferentes mecanismos dosadores. 1998. 86 f. Dissertação (Mestrado em Máquinas Agrícolas) - Escola Superior de Agricultura “Luiz de Queiroz”, Universidade de São Paulo, Piracicaba, 1998. 
DELAFOSSE, R.M. Máquinas sembradoras de grano gruesso. Santiago: Oficina Regional de La FAO para America Latina y el Caribe, 1986, 48 p.

EMBRAPA. Empresa Brasileira de Pesquisa Agropecuária. Sistema brasileiro de classificação de solos. Brasília: Embrapa Solos, 1999. 412 p.

FEY, E.; SANTOS, S.R.; FEY, A. Influência da velocidade de semeadura sobre a produtividade de milho (Zea mays L.). In: CONGRESSO BRASILEIRO DE ENGENHARIA AGRÍCOLA, 29., 2000, Fortaleza. Anais...Fortaleza: Sociedade Brasileira de Engenharia Agrícola, 2000. 1 CD.

HARRIGAN, T.M.; ROTZ, C.A. Draft of major tillage and seeding equipment. In: INTERNATIONAL WINTER MEETING, Atlanta, 1994. St Joseph: ASAE, 1994. 21 p. (Paper 941533).

KURACHI, S.A.H.; COSTA, J.A.S.; BERNARDI, J.A.; COELHO, J.L.O.; SILVEIRA, G.M. Avaliação tecnológica de semeadoras e/ou adubadoras: tratamento de dados de ensaios e regularidade de distribuição longitudinal de sementes. Bragantia, Campinas, v.48, n.2, p.249-62, 1989.

KURACHI, S.A.H.; SILVEIRA, G.M.; COSTA, J.A.; MORAES, R.A.D.M.; BERNARDI, J.A.; MOREIRA, C.A.; PETRONI, A.C.; SILVA, J.R.; MESQUITA, C.M. Código de avaliação de semeadoras e/ou adubadoras. Campinas: Instituto Agronômico de Campinas, 1986. v.3, 138 p. (Documentos)

MAHL, D.; GAMERO, C.A. Consumo no plantio. Cultivar Máquinas, Pelotas, n.22, p.18-21, 2003.

MAHL, D.; GAMERO, C.A.; BENEZ, S.H.; LEITE, M.A.S.; SILVA, A.R.B.; PONTES, J.R.V.; MARQUES, J.P.; GREGO, C.R.; COSTA, A.M. Distribuição longitudinal e profundidade de deposição de sementes de uma semeadora-adubadora de plantio direto em função da velocidade e mecanismo sulcador. In: CONGRESSO BRASILEIRO DE ENGENHARIA AGRÍCOLA, 30., 2001, Foz do Iguaçu. Anais... Foz do Iguaçu: Sociedade Brasileira de Engenharia Agrícola, 2001. 1 CD.

MARQUES, J.P.; PONTES, J.R.; BENEZ, S.H. Desempenho de uma semeadora-adubadora de precisão na semeadura de milho em preparo convencional do solo e em semeadura direta. In:

CONGRESSO BRASILEIRO DE ENGENHARIA AGRÍCOLA, 28., 1999, Pelotas. Anais... Pelotas: Sociedade Brasileira de Engenharia Agrícola, 1999. 1 CD.

OLIVEIRA, M.L. Avaliação do desempenho de uma semeadora-adubadora para plantio direto, em duas classes de solo com diferentes tipos de cobertura vegetal. 1997. 50 f. Dissertação (Mestrado em Engenharia Agrícola) - Universidade Federal de Viçosa, Viçosa - MG, 1997.

SILVA, J.G.; KLUTHCOUSKI, J.; SILVEIRA, P.M. Desempenho de uma semeadora-adubadora no estabelecimento e na produção da cultura do milho sob plantio direto. Scientia Agrícola, Piracicaba, v.57, n.1, p.7-12, 2000.

SILVA, S.L. Avaliação de semeadoras para plantio direto: demanda energética, distribuição longitudinal e profundidade de deposição de sementes em diferentes velocidades de deslocamento. 2000. 123 f. Tese (Doutorado em Energia na Agricultura) - Faculdade de Ciências Agronômicas, Universidade Estadual Paulista, Botucatu, 2000.

SIQUEIRA, R.; ARAÚJO, A.G.; CASÃO JÚNIOR, R.; RALISCH, R. Desempenho energético de semeadoras-adubadoras de plantio direto na implantação da cultura da soja (Glycine max L.). In: CONGRESSO BRASILEIRO DE ENGENHARIA AGRÍCOLA, 30., 2001, Foz do Iguaçu. Anais... Foz do Iguaçu: Sociedade Brasileira de Engenharia Agrícola, 2001. 1 CD. 